\title{
Nepali Language
}

National Cancer Institute

\section{Source}

National Cancer Institute. Nepali Language. NCI Thesaurus. Code C154051.

An Indo-Aryan language that is the official language of Nepal. 